\title{
In-situ and label-free optical monitoring of the adhesion and spreading of primary monocytes isolated from human blood: dependence on serum concentration levels
}

\author{
Norbert Orgovan $^{\text {a,b }}$, Rita Salánki ${ }^{\text {b,c }}$ Noémi Sándor $^{\mathrm{d}}$, Zsuzsa Bajtay ${ }^{\mathrm{e}}$, Anna Erdei ${ }^{\mathrm{d}, \mathrm{e}}$, \\ Bálint Szabó $^{\mathrm{a}}$ and Robert Horvath ${ }^{\mathbf{b}^{*}}$
}

${ }^{a}$ Department of Biological Physics, Eötvös Loránd University, Pázmány P. stny. 1/A, H-1117 Budapest, Hungary

${ }^{b}$ Nanobiosensorics Group, Hungarian Academy of Sciences, Research Centre for Natural Sciences, Institute for Technical Physics and Materials Science, Konkoly-Thege út 29-33, H-1120 Budapest, Hungary

${ }^{c}$ Doctoral School of Molecular- and Nanotechnologies, University of Pannonia, Veszprém, Hungary

${ }^{d}$ MTA-ELTE Immunology Research Group, ELTE, Pázmány P. stny. 1/C, H-1117 Budapest, Hungary

${ }^{e}$ Department of Immunology, Eötvös Loránd University, Pázmány P. stny. 1/C, H-1117 Budapest, Hungary

*email: horvathr@mfa.kfki.hu 


\section{Abstract}

Adhesion and spreading of primary monocytes isolated from human blood were monitored utilizing optical waveguide lightmode spectroscopy (OWLS); a highly sensitive label-free biosensor technique using evanescent optical waves generated at a biocompatible surface. Appropriate development on a custom built setup enabled the OWLS cuvette to be operated as a $1.5 \mathrm{ml}$ mini-incubator, controlling both temperature and $\mathrm{CO}_{2}$ levels. The incubator-equipped OWLS is readily applicable for delicate and long-term studies on sensitive primary cells, demonstrated here through monitoring the serum dependence of the adhesion and spreading of human monocytes. Moreover, the custom-built setup enables the simultaneous monitoring of the position and overall width of the OWLS resonant peaks. This unique feature makes it possible to distinguish the refractive index variations induced by the adsorption of secreted material from refractive index changes provoked by cellular spreading. A definite attachment and spreading activity was observed on the substratum (glassy silica-titania), when the serum level of the culturing medium was $0.0-0.01 \%$. Increasing serum concentration resulted in a steep fall in monocyte surface adhesion and spreading. $1.0 \%$ serum level practically abolished all spreading activity measured by OWLS, and the number of attached cells was significantly decreased, too. Serum addition to fully spread cells provoked a reduction in the cell-substratum contact area, clearly detectable by the biosensor. Cell spreading was inhibited by pre-coating the sensor surface with considerable amounts of serum proteins. These findings suggest that monocyte spreading is inhibited by the adsorption of serum biomolecules to the substratum, rather than by soluble factors present in the serum. All of these results were obtained completely non-invasively with real time monitoring; demonstrating the capabilities of OWLS to sensitively monitor the adhesion properties of immune cells isolated from human blood. 
The current study is, therefore, a significant step towards the application of label-free optical biosensors in medical diagnostics.

\section{Keywords:}

label-free optical biosensor

Optical Waveguide Lightmode Spectroscopy

mini-incubator

primary monocyte

cellular adhesion

serum dependence 


\section{Introduction}

Monocytes are a type of white blood cells (leukocytes) playing regulatory and effector roles, both of pivotal importance in innate immune function (Dale et al., 2008). Initially they reside in the blood and monitor foreign substances, but further roles require that monocytes leave the circulatory system by penetrating through the epithelium of the blood vessels and migrate to the neighboring tissues. These monocytes differentiate to dendritic cells (DCs) and macrophages (MFs) to either replenish the declining resident population of those cells under normal conditions or to give rise to a locally increased population of DCs and MFs, which determine the immune response at inflammation sites. Adhesion of monocytes to the endothelial cells (Beekhuzien and van Furth, 1993) and to components of the extracellular matrix $(\mathrm{ECM})$ is essential for the complex, multi-step process of transmigration (Ley et al., 2007). Compared to non-leukocytes, monocytes exhibit some unique features in their adhesion, which is associated with their function (Gahmberg, 1997; Harris et al., 2000; Ley et al., 2007; van Kooyk and Fidgor, 2000); they express leukocytespecific $\beta_{2}$ integrins, which can be rapidly activated upon stimulation (e.g. by inflammatory cytokines) (Gahmberg, 1997; Harris et al., 2000; Ley et al., 2007), and provoke the cell to undergo from weakly to highly adherent state.

Several diseases are associated with malfunctions in the tightly regulated adhesion of monocytes to the endothelium or to the ECM. Leukocyte adhesion deficiency (LAD) syndrome is characterized by insufficient leukocyte expression of $\beta_{2}$-integrins leading to life-threatening infections in patients (Anderson and Springer, 1987; Beekhuzien and van Furth, 1993). Monocyte adherence has been reported to be also impaired in patients with diabetes mellitus or alcohol-induced cirrhosis (Kelly et al., 1985), which is the reason why these patients are more prone to infections. Monocytes specifically adhering to damaged 
arterial endothelial cells and forming foam cells have major importance in the pathogenesis of atherosclerosis (Beekhuzien and van Furth, 1993; Huo and Ley, 2001).

Due to the extensive research of the last decades on leukocyte extravasation, much is known about the activators, mediators and regulators of the distinct steps of the leukocyte adhesion cascade (Ley et al., 2007). However, e.g., the complex regulatory function of the ECM on lymphocyte migration and its modulating role during the inflammatory immune response have gained attention only very recently (Korpos et al., 2010; Schor et al., 2000; Sorokin, 2010; Vaday and Lider, 2000).

In order to achieve a more detailed understanding of the mechanism of monocyte adhesion, we wish to move in the direction of increased precision in the quantification of the cells' behavior when they encounter the ECM. This precision should, ideally, be obtained in real time, and with negligible perturbation of the system. These criteria can be met by using a high-resolution label-free biosensing technique, optical waveguide lightmode spectroscopy (OWLS) (Ramsden, 1998).

OWLS is a high performance surface-sensitive technique allowing label-free real-time monitoring of processes accompanied by refractive index changes in the close vicinity of a waveguiding sensor chip (a thin waveguiding layer supported on a thicker optical glass slide and having a shallow diffraction grating embedded into the structure). The successful incoupling of a linearly polarized monochromatic laser beam via the grating is characterized by a sharp resonance peak when plotting the intensity of the incoupled light against the incident angle of the illuminating beam. The sensing principle is based on the perturbation of 
the evanescent waves of the guided lightmodes; refractive index variations alter the discrete incoupling angles so the position of the resonance peak will be shifted (Tiefenthaler and Lukosz, 1989; Vörös et al., 2002).

In the present OWLS configuration (the so-called conventional configuration in which the refractive index of the substratum is greater than that of the covering medium) the evanescent field penetrates to a 100-200 $\mathrm{nm}$ thick layer above the planar waveguide, making OWLS suitable for monitoring the contact area of living cells (Aref et al., 2010a, 2010b; Hug et al., 2002; Ramsden et al., 1994). Using reverse symmetry waveguides (Horvath et al., 2002), supported on substrata with lower refractive index than that of the covering medium, the penetration depth of the evanescent field can be increased and fine-tuned (Horvath et al., 2008, 2005b, 2003b), making it possible to monitor changes deeper inside cells (Horvath et al., 2008). Another advantage of OWLS over other sensing platforms (Ramsden, 1997) is that it enables kinetic readout of multiple parameters characterizing the OWLS's resonance peaks (Cottier and Horvath, 2008). Comparative analysis of the temporal evolution of these parameters enables to distinguish the refractive index variations caused by secreted molecules from refractive index changes provoked by cellular spreading (Ramsden and Horvath, 2009). Noted that the substratum is the high refractive index waveguiding film, typically about 200 $\mathrm{nm}$ thick, for which a wide range of biocompatible materials (e.g., titania, niobia (Starikov et al., 2007)) are available. Moreover, these substrata can be easily surface-modified or coated with a thin layer of virtually any material, provided it is transparent at the wavelength of the guided light. This gives a tremendous flexibility to the technique.

Given that high sensitivity, excellent temporal resolution, and label-free detection principle are further characteristics of the technique, we believe that OWLS has an outstanding 
potential to contribute to a deeper understanding of monocyte adhesion. Although OWLS has previously been used to investigate the adhesion and spreading of various cell lines (Aref et al., 2010a, 2010b; Horvath et al., 2008, 2005a; Hug et al., 2002, 2001; Li et al., 1994; Ramsden and Horvath, 2009; Ramsden et al., 1994), its potentials have never hitherto been exploited in primary cell studies.

The purpose of this paper is twofold. Firstly, we describe a significant instrumental development of a previously constructed (Horvath et al., 2008, 2003a, 2002) OWLS setup. We developed the OWLS sample cuvette into a mini-incubator that enables control over the temperature and atmosphere of the living cell environment while retaining all the other convenient functions. This upgraded OWLS can be readily used for the long term monitoring of sensitive cells. Secondly, we utilized this incubator-equipped setup to characterize monocyte adhesion and spreading as a function of the serum content of the culture medium. Analysis of the OWLS data enables the underlying cause of the observed dependence now be understood in its details.

Beyond these advances, we consider these experiments as the next step towards medical diagnostic applications, especially those involving the monitoring of primary cells noninvasively.

\section{Materials and methods}

\subsection{Sensor chip cleaning and pretreatment}

Prior to experiments, the applied OW2400 waveguide sensor chip (Microvacuum Ltd., Hungary) underwent a cleaning routine. First, they were immersed into chromic acid (Merck) 
for 3 minutes, followed by subsequent rinsing with Milli-Q water (MQ), potassium hydroxide $(\mathrm{KOH})$, and MQ again. Afterwards the chips were placed into an ultrasonicator for at least 30 minutes and the bathing MQ water was changed every 3 minutes over them. Prior to experiments the cleaned waveguides were incubated in serum-free Roswell Park Memorial Institute (RPMI, Sigma) medium overnight.

\subsection{Monocyte isolation from human blood}

Peripheral blood mononuclear cells (PBMCs) were isolated from buffy coat obtained from healthy donors and provided by the Hungarian National Blood Transfusion Service by density gradient centrifugation on Ficoll-Paque (GE Healthcare). Informed consent was provided for the use of blood samples according to the Declaration of Helsinki. Unlabeled monocytes were isolated by negative magnetic separation using the Miltenyi Monocyte Isolation kit II (Miltenyi) according to the manufacturer's instructions. In short, nonmonocytes are indirectly magnetically labeled using a cocktail of biotin-conjugated antibodies and anti-biotin MicroBeads. Highly enriched unlabeled monocytes are obtained by depletion of the magnetically labeled cells. Cells were cultivated in RPMI-10\% FBS $\left(37^{\circ} \mathrm{C}, 5 \% \mathrm{CO}_{2}\right.$ atmosphere) media in Teflon coated flasks to avoid spontaneous monocyte attachment to the culture dish. OWLS experiments were carried out within one day after their isolation to exclude the spontaneous differentiation into MFs or DCs.

\subsection{Cell spreading assay on the OWLS sensor}

Assay media for the OWLS experiments were prepared by adding $4 \mathrm{mM}$ glutamine, $40 \mu \mathrm{g} / \mathrm{ml}$ gentamicin, $0.25 \mu \mathrm{g} / \mathrm{ml}$ amphotericin-B, and fetal bovine serum (FBS) in the desired volume to the RPMI medium (all substances were obtained from Sigma-Aldrich). 
OWLS measurements were initiated by taking a baseline with $1400 \mu \mathrm{l}$ of assay medium, while both the temperature $\left(37{ }^{\circ} \mathrm{C}\right)$ and $\mathrm{CO}_{2}$-level $(5 \%)$ were controlled with the novel miniincubator. Monocytes were centrifuged at $300 \mathrm{~g}$ for $7 \mathrm{~min}$, and gently suspended in $1 \mathrm{ml}$ fresh assay medium. Cells were then counted with a hemocytometer. At this point, the OWLS scanning was stopped and, if not stated otherwise, 60,000 cells were pipetted into the cuvette having a bottom surface area of $44 \mathrm{~mm}^{2}$. Then the scanning was continued, and the enclosed atmosphere was renewed with $5 \% \mathrm{CO}_{2}$ by opening the valve manually for $10-15$ seconds.

During the $2 \mathrm{~h}$ long spreading measurements, $5 \% \mathrm{CO}_{2}$-atmosphere was sustained by automatic injections $(5 \mathrm{~s} / 10 \mathrm{~min})$ from a tank.

\subsection{Visualization of the cell-substratum contact area and image analysis}

At the end of the OWLS experiments the assay medium was carefully removed from the cuvette with a pipette, unspread cells were washed away by gently pipetting phosphate buffered saline (PBS, Sigma) onto the sensor disassembled from the cuvette, and finally, cells remaining on the sensor were fixed with paraformaldehyde (4\% PFA, 20 min). Fixed cells were visualized with an inverted Zeiss Axio Observer A1 microscope using a 10x objective, and a representative phase contrast image was taken. Mean cell-substratum contact areas were accessed by tracking the contour of 50 cells in each image and averaging the enclosed contact areas (measured using ImageJ).

\subsection{Cell viability}

The viability of cells that spread on the sensor surface for two hours in the total absence of serum was checked with a trypan blue test, and all spread cells were found to be fully viable. 


\section{Results and discussion}

\subsection{Mini-incubator development for the OWLS setup}

To avoid disturbing effects of temperature or $\mathrm{pH}$ fluctuations on the OWLS signal, and also to better mimic in vivo conditions, we needed to control the temperature and $\mathrm{CO}_{2}$ levels in the OWLS cuvette. This required further developments on our custom built OWLS setup (Horvath et al., 2008, 2003a, 2002). The cuvette of the improved OWLS system can be temperature stabilized at $37^{\circ} \mathrm{C}$. The heating module is attached to the back of the track in which the cuvette holder can be fastened. Both the track and the cuvette holder are made of metal and small in size, thus heat is transmitted very effectively towards the waveguide sensor (which also serves as the bottom of the cuvette and the substratum for adhering cells). We verified that the sensor measures the temperature of the assay medium with a precision of $0.1{ }^{\circ} \mathrm{C}$. The optical unit of the OWLS (the high-precision goniometer holding the cuvette and the photodiodes on both sides of the sensor) was heat insulated by enclosing it in a polystyrene foam box. In this way, we obtained temperature control of the cuvette with a precision of $\pm 0.1^{\circ} \mathrm{C}$.

The $\mathrm{pH}$ of the assay medium in the OWLS cuvette is maintained by an appropriate $\mathrm{CO}_{2}$ atmosphere. Special gas mixture containing $5 \% \mathrm{CO}_{2}$ is infused with a preset periodicity and duration through a valve operated by an external, programmable control unit (see Fig. 1). The valve and the cuvette are connected with a Teflon tube. The distal end of this tube is introduced into a cap which can be tightly pulled over the top of the closed cuvette. In order to minimize the disturbing effects of direct gas infusion on the biosensor signal, a plug with a gap of $0.1 \mathrm{~mm}$ around its cylinder is inserted between the cap and the assay 
medium, thus gas directly above the medium is exchanged by diffusion rather than by flow. Overpressure is guided away from the cuvette by an additional tube with its other end kept under water to avoid the backflow of air. A photo showing the assembled miniincubator cuvette is presented in the inset of Fig. 1.

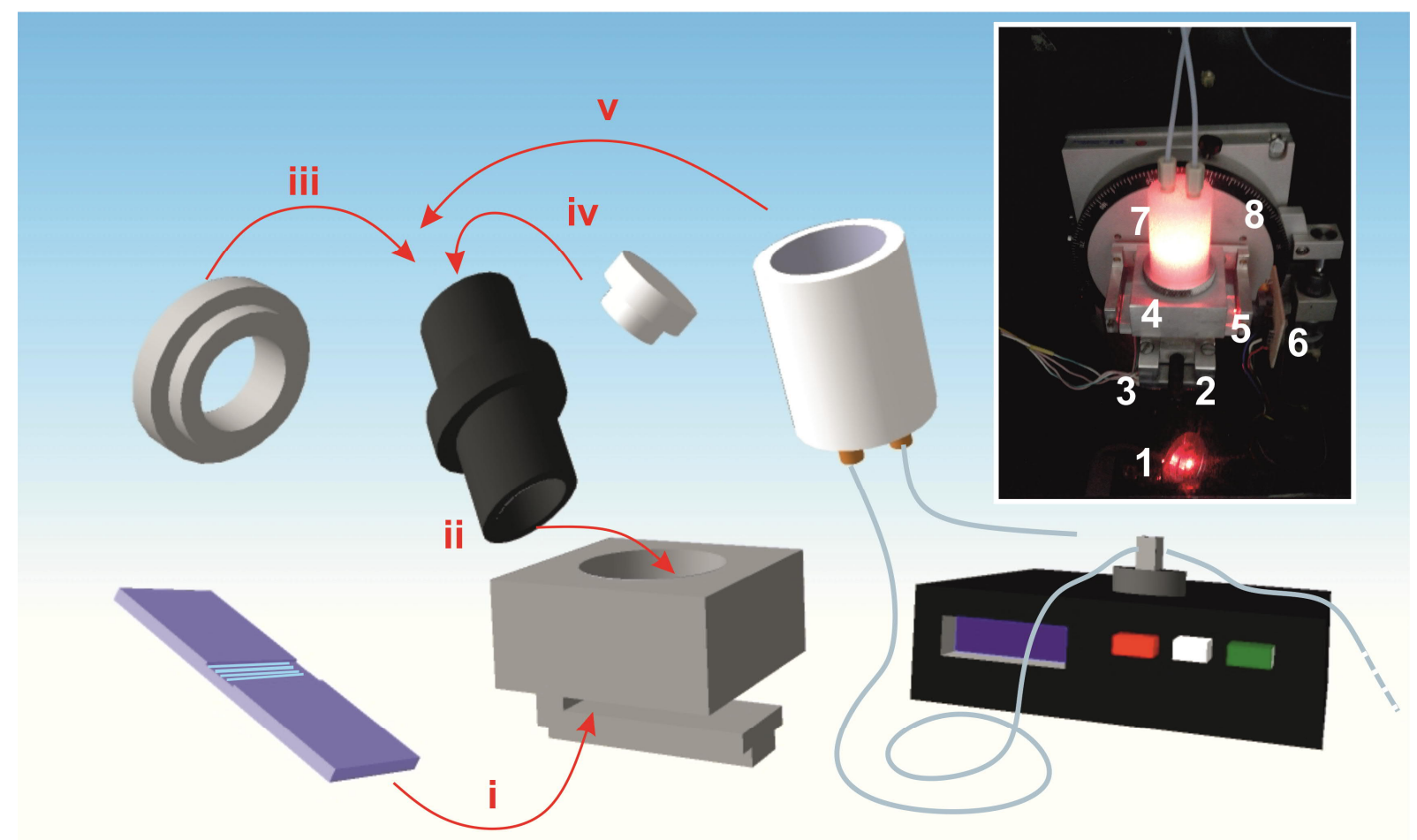

Figure 1.

\subsection{OWLS measurements on primary monocytes}

The incubator-equipped OWLS setup was utilized to investigate the dependence of monocyte adhesion and spreading on the serum content of the culture medium.

Inset in Fig 2a depicts how the overall shape (central position $\alpha$, and overall width $\delta$ (Cottier and Horvath, 2008)) of the transverse magnetic (TM) resonance peak is altered during a typical cell adhesion experiment. The position of the peak is shifted to higher incident angles due to refractive index increment near the surface, which can originate from cell spreading and/or surface adsorption of biomolecules secreted by the cells 
(Ramsden and Horvath, 2009). On the other hand, width of the resonance peak reflects the extent of micrometer scale inhomogeneity in the refractive index, thus its variation is a specific indicator of cell spreading, and is not affected by any adsorbing biomolecules (Cottier and Horvath, 2008; Ramsden and Horvath, 2009). Numerical simulations have proven that maximum width corresponds to the case when $50 \%$ of the surface is covered by cells (Cottier and Horvath, 2008).
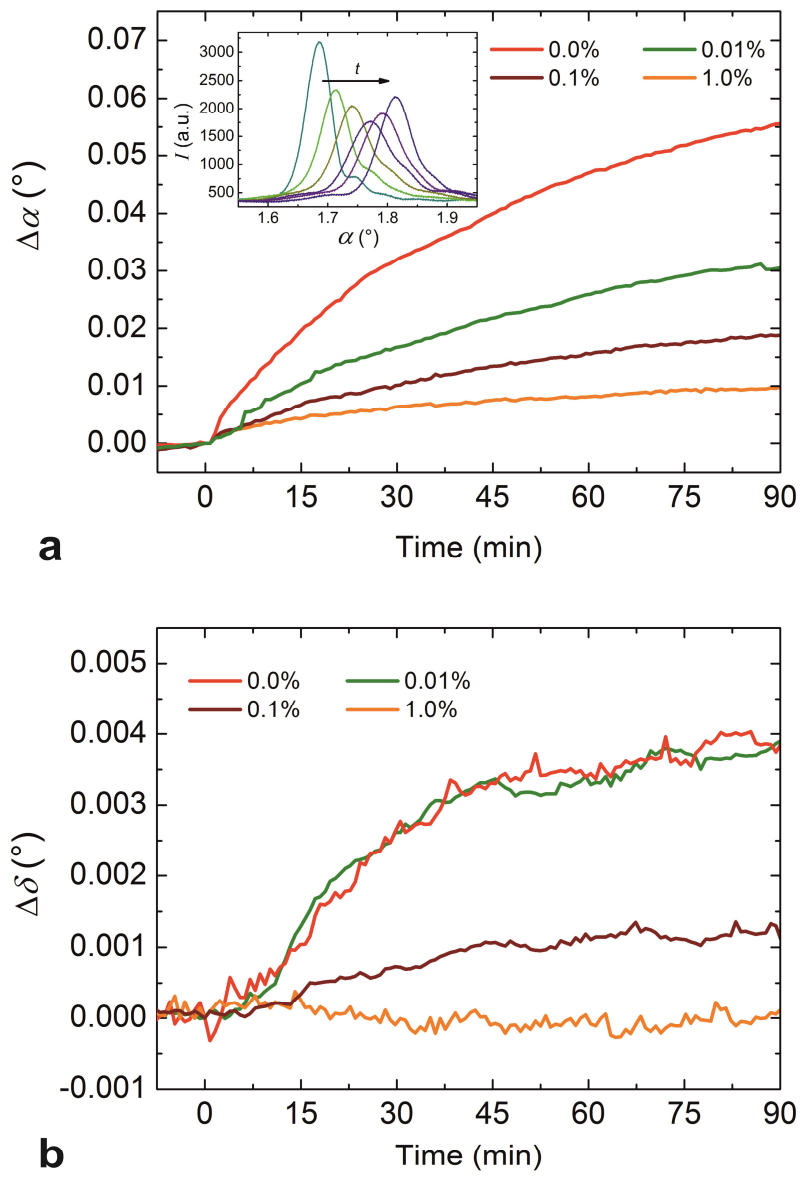

Figure 2.

Panels (a) and (b) in Fig. 2 show representative kinetic data for these two parameters, i.e., $\alpha$ and $\delta$, measured on the same day by monitoring the adhesion of monocytes in the presence of the indicated serum concentrations $(0.0 \%, 0.01 \%, 0.1 \%$ and $1.0 \%$ FBS $)$. We 
found that FBS above the concentration of $1.0 \%$ abolished practically all spreading activity detectable by OWLS. Monocytes seeded in the presence of 1.0\% FBS induced a slight shift in $\alpha$ over time (Fig. 2a), while no change was observed in $\delta$ (Fig. 2b). These findings may be explained by the adsorption of molecules secreted by the cells rather than by cell spreading (Aref et al., 2010a). This hypothesis is further strengthened by the non-sigmoidal shape of the peak position vs. time curves (Aref et al., 2010a; Ramsden and Horvath, 2009). When decreasing the FBS concentration, the peak position vs. time curves increased monotonically (Fig. 2a). In contrast, the peak width $v s$. time curves increased with decreasing serum concentration and reached a maximum at $0.01 \% \mathrm{FBS}$, i.e., the signal did not increase further when decreasing the serum content to $0.0 \%$ (see Fig $\mathbf{2 b}$ ).

We replotted $\Delta \delta$ against $\Delta \alpha$ to elucidate the origin of these findings in more detail (Fig. 3 ). Interestingly, the same shift in $\alpha$ was accompanied by a greater $\Delta \delta$ when the assay medium contained $0.01 \%$ FBS as compared to $0.0 \%$ FBS. This strongly suggests that the most intensive spreading activity took place at intermediate serum dilutions $(\sim 0.01 \%)$. When the serum content was decreased from $0.01 \%$ to $0.0 \%$, only $\alpha$ increased further, while $\delta$ followed the same evolution as in the presence of 0.01\% FBS (Fig. 2ab). This suggests that more secreted material adsorbed to the bare surface (which did not receive any serum) - either due to an increased secretion activity and/or the highest ratio of the surface being available for adsorbing species. 


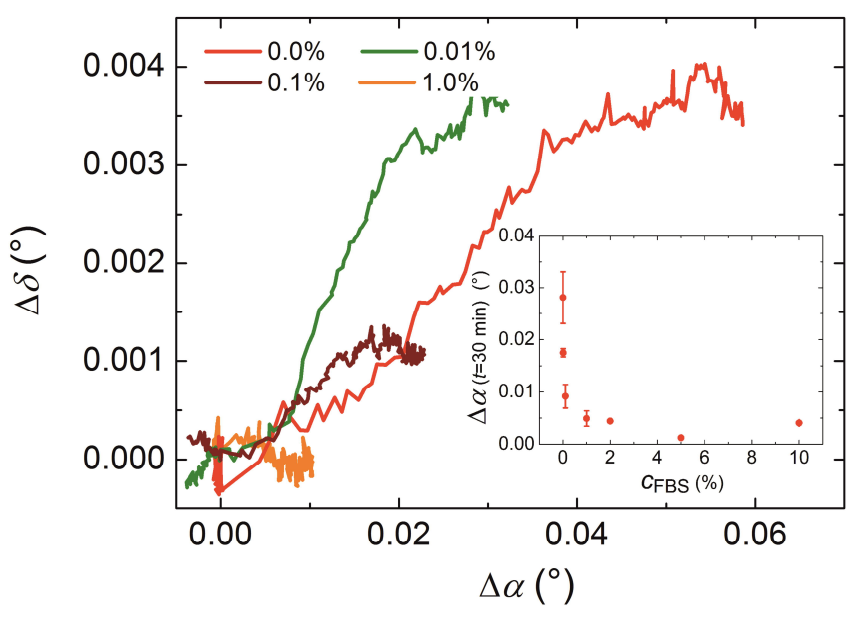

Figure 3.

We mapped the serum dependence of monocyte adhesion at a higher resolution (Fig. 3 inset). Here cellular activity is characterized by the OWLS signal $\Delta \alpha$ that was obtained 30 min after seeding of the cells. Each point on the graph is the mean of the results of at least duplicates of experiments. These results confirm our previous findings, as the inset in Fig. $\mathbf{3}$ shows an exceptionally strong dependence of spreading on the serum content. Drastically less cells attached and spread when the serum level was increased, which might be associated with the non-adherent state of quiescent monocytes described in vivo (Dale et al., 2008).

We hypothesized that the spreading of monocytes was inhibited by proteins adsorbed from the serum rather than by soluble factors in it. This was tested in two separate OWLS experiments; in these $10^{5}$ cells were seeded in order to obtain larger biosensor responses. First, we added serum to cells which have previously spread in its absence. This triggered a steady robust decrease in $\alpha$ (Fig. 4 main image), which was the result of cell retraction and serum protein adsorption, the former being the dominant. The width of the resonance peak vs. time (inset in Fig. 4) confirms that cells retracted from the surface in a response to 
serum, as the degree of inhomogeneity derivable from the optical width (Cottier and Horvath, 2008), started to increase again (solid red curve). In comparison, cells seeded in the presence of $20 \%$ serum did not show any spreading activity (dashed blue curves on Fig. 4). These results suggest that spreading is demolished by the serum proteins adsorbed to the surface, especially that of the cell-anti-adhesive albumin, the most abundant protein in serum. This was further confirmed in the second experiment, where cells were seeded in the absence of serum onto a sensor surface that has been pre-coated with $10 \%$ serum, and no spreading activity was observed (data not shown).

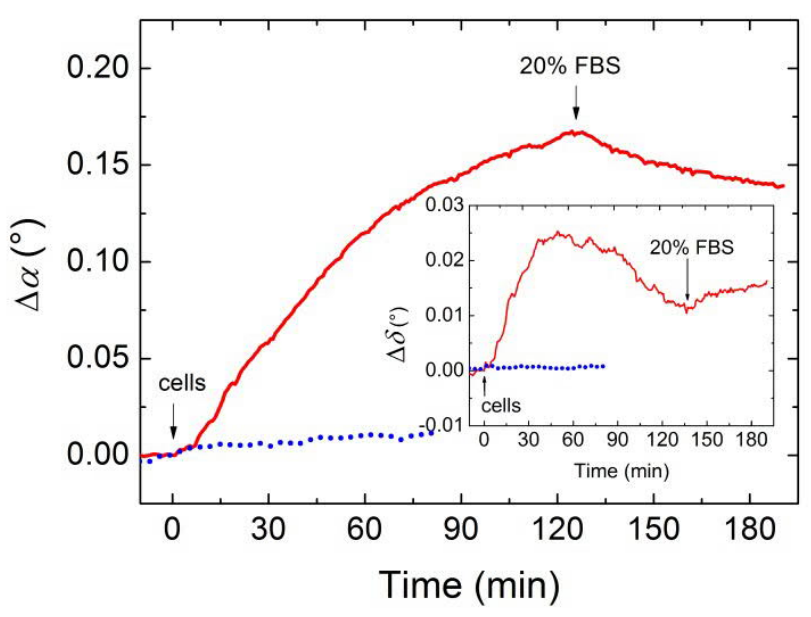

Figure 4.

\subsection{Analysis of phase-contrast images of cells spread on the optical waveguide}

At the end of each of the four experiments discussed above (Fig. 2 and 3), cells were visualized with a phase contrast microscope. Representative images are presented in Fig. $\mathbf{5}$. 
Analysis performed on the fields-of-view images facilitated the appropriate interpretation of the biosensor data. Based on the observed morphologies, cells were classified and ordered into four subgroups (Fig. 6). Type 1, appearing on the images with a dark cell body and a well-established peripheral skirt of cytoplasm around it, is the most spread type. Type 2 attains a similar, often elongated morphology, but does not have a skirt around the main cell body. In contrast, a Type 3 cell, showing a rounded morphology and high brightness, is weakly adhered or even floating. Type 4 is an aggregate of at least five Type 3 cells. Cells that have attained distinct morphologies were counted separately in the fields-of-view images. Resulting statistics for cell populations spread at various serum concentrations $(0.0 \%, 0.01 \%, 0.1 \%$ and $1.0 \%$ FBS $)$ are shown in Fig. 6. The contribution of Type 3 and Type 4 cells to the biosensor signal, especially to $\Delta \delta$, is negligible compared to that of Type 1 and Type 2 cells (spread types) (Aref et al., 2010a). As Fig. 6 indicates, the summed number of Type 1 and Type 2 cells are nearly equal for $0.0 \%$ and $0.01 \%$ FBS content in the assay medium. In contrast, the number of spread-type cells decreases drastically and monocytes tend to form aggregates when the FBS content of the culture medium is further increased.

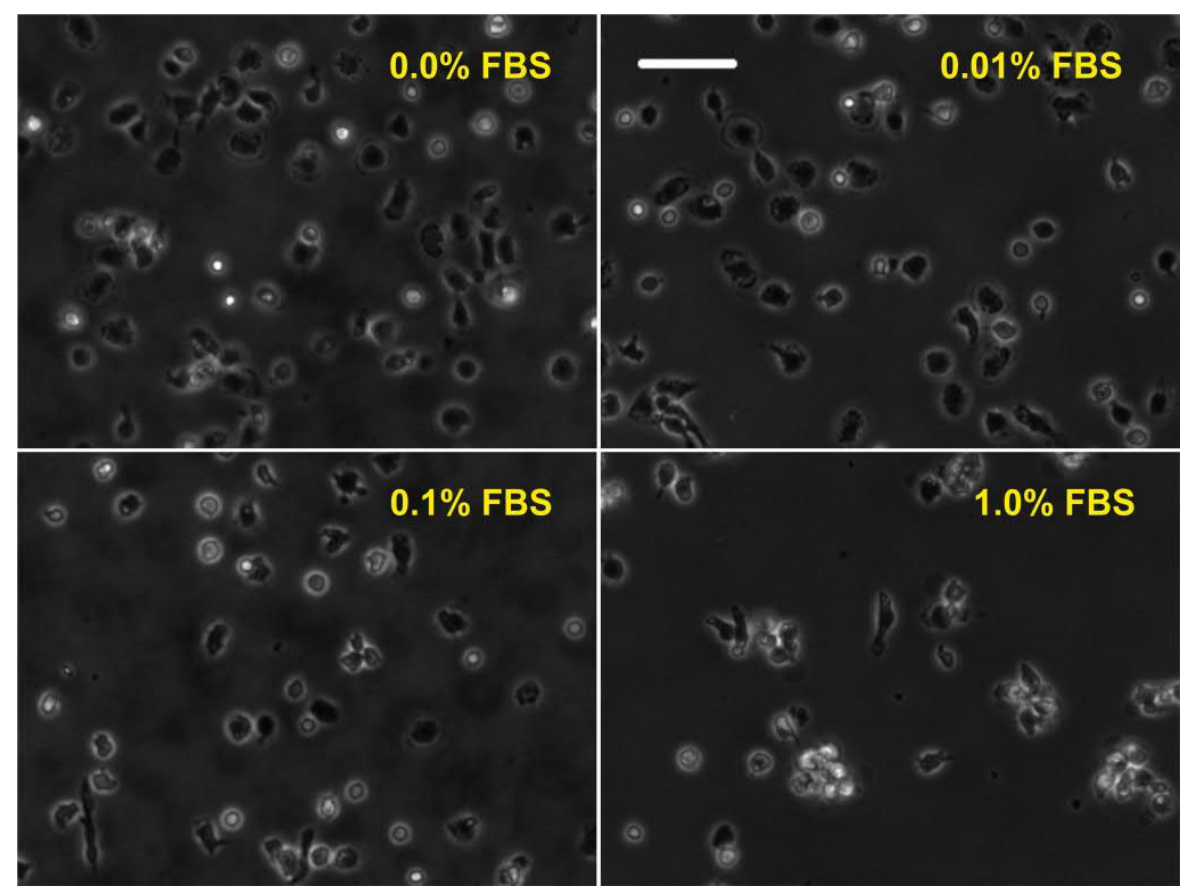


Fig. 5

The $A_{c_{\mathrm{FBS}}}$ mean contact area (expressed as mean \pm 'standard error of the mean') of cells spread in the presence of various serum concentrations $\left(c_{\mathrm{FBS}}\right)$ was measured to be as follows: $A_{0.0 \%} \exists 37 \sharp 4 \quad, A_{0.01 \%} \geq 38 \sharp 2 \quad, A_{0.1 \%} \neq 45 \pm \quad, A_{1.0 \%} \neq 08 \pm$ $5 \mu \mathrm{m}^{2}$. These results evidence that both the number of spread-type cells and their average contact area are decreasing with increasing serum concentration. Compared with the spreading in the absence of serum, the total area covered by cells (which is the product of the number of spread-type cells and their mean contact area) decreased by a factor of 2.77 , while the end-point value of $\Delta \delta$ decreased by a factor of 3.17 (Fig. 2b) when $0.1 \%$ FBS was present in the assay medium. Therefore, we conclude that the findings obtained from image analysis are in nice accordance with the OWLS data, and support its interpretation described in $\S 3.2$. 


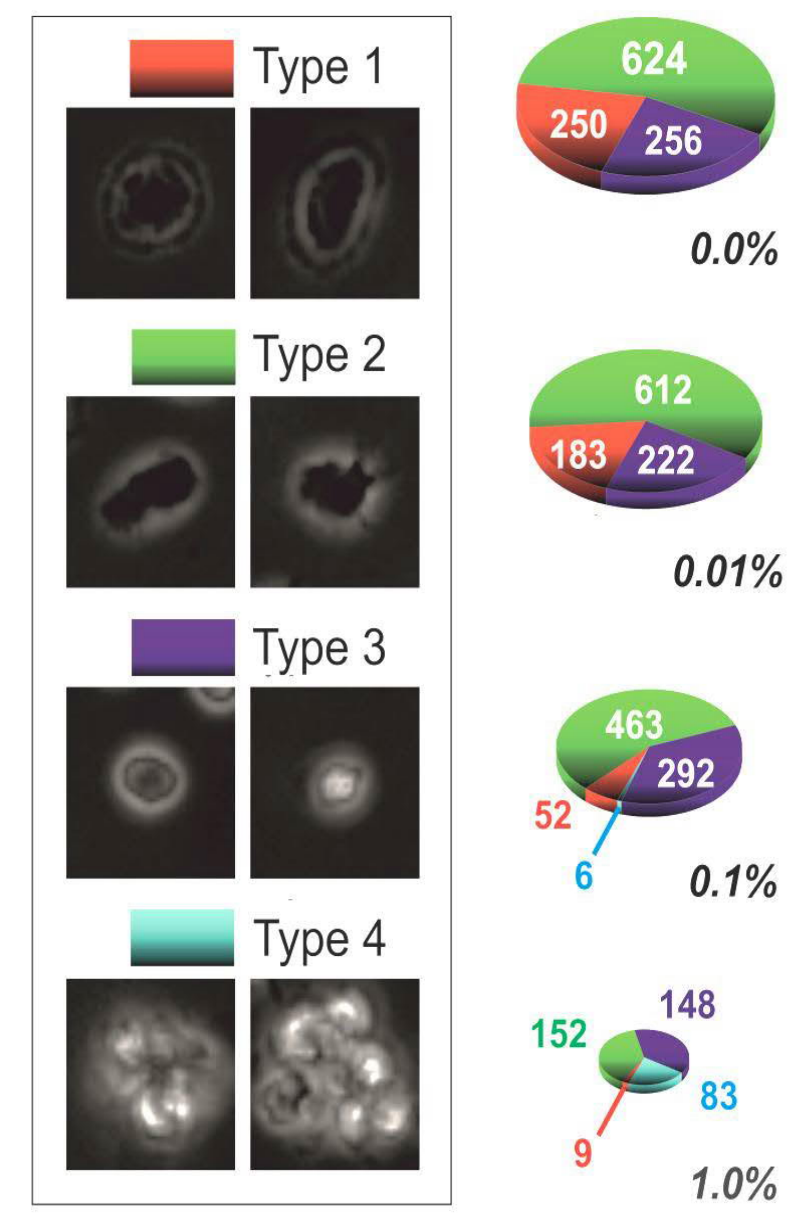

Fig. 6.

\section{Conclusions}

The present report is the first to demonstrate that "zero-perturbation" OWLS is suitable for monitoring the adhesion and spreading of primary cells. In particular, we consider that the use of OWLS to characterize cells freshly isolated from human blood is a crucial step towards the application of optical biosensors to medical diagnostics. Such cells require delicate handling (including, preferably, the avoidance of labelling) and culture conditions approximating those they have in vivo. To ensure the latter, we successfully developed a sample cuvette that also functions as a mini-incubator while retaining all its other functions such as the possibility of pipetting diverse solutions over the cells. 
The novel setup was applied to investigate the adhesion and spreading of human monocytes on a silica-titania substratum; here we elucidated the serum dependence of the process. A definite attachment and spreading activity was observed on the sensor surface when the culturing medium contained no serum. Cells seeded in the presence of $0.01 \%$ serum spread identically with those spreading in serum-free medium, but a less marked surface adsorption of secreted material was detected in the former case. Further increasing serum concentration resulted in a steep fall in monocyte cell adhesion. $1.0 \%$ serum practically abolished all spreading activity measured by OWLS, and the number of attached cells was significantly decreased, too. The addition of serum to cells that have previously spread in its absence caused the cell-substratum contact area to significantly diminish. We infer that the observed inhibitory effect of serum is due to the adsorption of cell-anti-adhesive serum biomolecules to the substratum.

It is important to stress that our results were obtained noninvasively without the incorporation of any labels (such as organic or nanoparticulate fluorophores). These advantages apply to other kinds of optical biosensors, albeit that they are typically less sensitive and less informative than OWLS. The principle of the mini-incubator could be applied to many different kinds of biosensors. OWLS also has the versatility to permit the cell substratum to be readily modified. Hence, the present approach could be applied to study the adhesion of other primary cells to a wide diversity of biological coatings and for monitoring the effects of receptor-specific mediators. 


\section{Acknowledgements}

We thank Jeremy Ramsden for critical comments on our work and for continuously supporting our OWLS activity. The present work was supported by the "Lendület" Program of the Hungarian Academy of Sciences and by the K104838 OTKA grant. 


\section{Figure captions}

Figure 1. Simple modification on our custom built OWLS setup allowed us to operate the OWLS cuvette as a mini-incubator. In this drawing only the parts enabling $\mathrm{CO}_{2}$ control are depicted. Roman numbers indicate the order of assembly: i-ii) the waveguide (sensor chip with optical grating) and the cuvette is inserted to the holder, iii) the cuvette is fixed with a screw, then iv) plugged and v) capped, the cap being connected to the $\mathrm{CO}_{2}$-infusion control unit (bottom left corner).

Inset: photograph of the assembled mini-incubator OWLS cuvette mounted on the goniometer. 1: mirror reflecting the laser light, 2: track into which the cuvette holder can be fixed, 3: heating module fastened to the back of the metallic cuvette holder, 4: cuvette holder, 5: sensor chip guiding the in-coupled light, 6: photodiode measuring the intensity of incoupled light, 7: cap for $\mathrm{CO}_{2}$-infusion, 8: high-precision goniometer.

Figure 2. OWLS responses provoked by monocyte adhesion and spreading in the presence of various serum concentrations (expressed as \% FBS dilution). All of the four experiments were carried out on the same day with monocytes of the same donor. Panel (a): shift of the position of the resonance peak $(\Delta \alpha)$ as a function of time. Inset: temporal evolution of the overall shape of the resonance peak. Panel (b): temporal evolution of the width of the resonance peak provoked by monocyte spreading in the presence of the indicated serum concentrations.

Figure 3. Alteration in the width of the resonance peak ( $\Delta \delta$ from Fig 2b) replotted against the shift in the peak position ( $\Delta \alpha$ from Fig 2 a) enables more detailed data analysis. Inset: the serum concentration $\left(c_{\mathrm{FBS}}\right)$ dependence of monocyte adhesion characterized by $\Delta \alpha$ obtained after $30 \mathrm{~min}$ of seeding. Each point on the graph is the mean of at least duplicated experiments. 
Figure 4. Serum addition to monocytes that have previously spread in the absence of serum triggers the cell-substratum contact area to retract. Solid red curves show the spreading in the absence of serum; at $t \notin 27$ min FBS was added to the medium to obtain an FBS concentration of $20 \%$. As a control, dashed blue curves reflect the abolished spreading activity in the presence of $20 \%$ FBS. Main image shows the shift in the position of the transverse magnetic resonance peak $(\Delta \alpha)$. Inset: alterations of the width $(\Delta \delta)$ of the TM resonance peak registered in the same experiments.

Figure 5. Representative phase contrast images of cultures fixed at the end of the OWLS experiments presented in Fig. 2. White scale bar on the second panel represents $50 \mu \mathrm{m}$.

Figure 6. Cells were classified based on their morphologies observed at the end of the OWLS experiments (Fig. 2). Microscope images to the right show two typical examples for each morphology (type). Cells belonging to the distinct types were counted for each experimental condition (varying FBS concentrations), and the resulting statistics is shown in pie charts (to the right). Relative size of the pie charts correlates with the total number of cells counted in the presence of the indicated serum concentrations (in italics). 


\section{References}

Anderson, D.C., Springer, T.A., 1987. Leukocyte adhesion deficiency: an inherited defect in the Mac-1, LFA-1, and p150,95 glycoproteins. Annu. Rev. Med. 38, 175-194.

Aref, A., Horvath, R., McColl, J., Ramsden, J.J., 2010a. Optical monitoring of stem cellsubstratum interactions. J. Biomed. Opt. 14, 010501.

Aref, A., Horvath, R., Ramsden, J.J., 2010b. Spreading kinetics for quantifying cell state during stem cell differentiation. J. Biol. Phys. Chem. 10, 1-7.

Beekhuzien, H., van Furth, R., 1993. Monocyte adherence to human vascular andothelium. Jotunal Leukoc. Biol. 54, 363-378.

Cottier, K., Horvath, R., 2008. Imageless microscopy of surface patterns using optical waveguides. Appl. Phys. B 91, 319-327.

Dale, D.C., Boxer, L., Liles, W.C., 2008. The phagocytes: neutrophils and monocytes. Blood $112,935-45$.

Gahmberg, C.G., 1997. Leukocyte adhesion: CD11/CD18 integrins and intercellular adhesion molecules. Curr. Opin. Cell Biol. 9, 643-50.

Harris, E.S., McIntyre, T.M., Prescott, S.M., Zimmerman, G. a, 2000. The leukocyte integrins. J. Biol. Chem. 275, 23409-12.

Horvath, R., Cottier, K., Pedersen, H.C., Ramsden, J.J., 2008. Multidepth screening of living cells using optical waveguides. Biosens. Bioelectron. 24, 805-10.

Horvath, R., Fricsovszky, G., Papp, E., 2003a. Application of the optical waveguide lightmode spectroscopy to monitor lipid bilayer phase transition. Biosens. Bioelectron. $18,415-28$.

Horvath, R., Pedersen, H.C., Larsen, N.B., 2002. Demonstration of reverse symmetry waveguide sensing in aqueous solutions. Appl. Phys. Lett. 81, 2166.

Horvath, R., Pedersen, H.C., Skivesen, N., Selmeczi, D., Larsen, N.B., 2003b. Optical waveguide sensor for on-line monitoring of bacteria. Opt. Lett. 28, 1233-5.

Horvath, R., Pedersen, H.C., Skivesen, N., Selmeczi, D., Larsen, N.B., 2005a. Monitoring of living cell attachment and spreading using reverse symmetry waveguide sensing. Appl. Phys. Lett. 86, 071101.

Horvath, R., Pedersen, H.C., Skivesen, N., Svanberg, C., Larsen, N.B., 2005b. Fabrication of reverse symmetry polymer waveguide sensor chips on nanoporous substrates using dipfloating. J. Micromechanics Microengineering 15, 1260-1264. 
Hug, T.S., Prenosil, J.E., Maier, P., Morbidelli, M., 2002. Optical waveguide lightmode spectroscopy (OWLS) to monitor cell proliferation quantitatively. Biotechnol. Bioeng. 80, 213-21.

Hug, T.S., Prenosil, J.E., Morbidelli, M., 2001. Optical waveguide lightmode spectroscopy as a new method to study adhesion of anchorage-dependent cells as an indicator of metabolic state. Biosens. Bioelectron. 16, 865-74.

Huo, Y., Ley, K., 2001. Adhesion molecules and atherogenesis. Acta Physiol. Scand. 173, $35-43$.

Kelly, M.K., Brown, J.M., Thong, Y.H., 1985. Neutrophil and monocyte adherence in diabetes mellitus, alcoholic cirrhosis, uraemia and elderly patients. Int. Arch. Allergy Appl. Immunol. 78, 132-138.

Korpos, E., Wu, C., Song, J., Hallmann, R., Sorokin, L., 2010. Role of the extracellular matrix in lymphocyte migration. Cell Tissue Res. 339, 47-57.

Ley, K., Laudanna, C., Cybulsky, M.I., Nourshargh, S., 2007. Getting to the site of inflammation: the leukocyte adhesion cascade updated. Nat. Rev. Immunol. 7, 678-89.

Li, S.Y., Ramsden, J.J., Prenosil, J.E., Heinzle, E., 1994. Measurement of adhesion and spreading kinetics of baby hamster kidney and hybridoma cells using an integrated optical method. Biotechnol. Prog. 10, 520-4.

Ramsden, J.J., 1997. Optical biosensors. J. Mol. Recognit. 10, 109-20.

Ramsden, J.J., 1998. Towards zero-perturbation methods for investigating biomolecular interactions. Colloids Surfaces A Physicochem. Eng. Asp. 141, 287-294.

Ramsden, J.J., Horvath, R., 2009. Optical biosensors for cell adhesion. J. Recept. Signal Transduct. Res. 29, 211-23.

Ramsden, J.J., Li, S.Y., Prenosil, J.E., Heinzle, E., 1994. Kinetics of adhesion and spreading of animal cells. Biotechnol. Bioeng. 43, 939-45.

Schor, H., Vaday, G.G., Lider, O., 2000. Modulation of leukocyte behavior by an inflamed extracellular matrix. Dev. Immunol. 7, 227-38.

Sorokin, L., 2010. The impact of the extracellular matrix on inflammation. Nat. Rev. Immunol. 10, 712-23.

Starikov, V. V, Starikova, S.L., Mamalis, A.G., Lavrynenko, S.N., Ramsden, J.J., 2007. The application of niobium and tantalum oxides for implant surface passivation 7, 141-145.

Tiefenthaler, K., Lukosz, W., 1989. Sensitivity of grating couplers as integrated-optical chemical sensors. J. Opt. Soc. Am. B 6, 209.

Vaday, G.G., Lider, O., 2000. Extracellular matrix moieties, cytokines, and enzymes: dynamic effects on immune cell behavior and inflammation. J. Leukoc. Biol. 67, 149-59. 
Van Kooyk, Y., Fidgor, C.G., 2000. Avidity regulation of integrins : the driving force in leukocyte adhesion. Curent Opin. Cell Biol. 12, 542-547.

Vörös, J., Ramsden, J.J., Csúcs, G., Szendrő, I., De Paul, S.M., Textor, M., Spencer, N.D., 2002. Optical grating coupler biosensors. Biomaterials 23, 3699-3710. 